\title{
Why is poverty unhealthy? Social and physical mediators
}

\author{
Deborah A. Cohen ${ }^{\mathrm{a}, \mathrm{b}, *}$, Thomas A. Farley ${ }^{\mathrm{c}}$, Karen Mason ${ }^{\mathrm{b}}$ \\ ${ }^{a}$ RAND Corporation, 1700 Main Street, 2138, Santa Monica, CA, USA \\ ${ }^{\mathrm{b}}$ Louisiana State University Health Science Center, USA \\ ${ }^{\mathrm{c}}$ Tulane University School of Public Health and Tropical Medicine, USA
}

\begin{abstract}
Socioeconomic status is associated with mortality, yet does not fully explain health disparities. This study analyzed data from the Project on Human Development in Chicago Neighborhoods (PHDCN), in the USA, to identify neighborhood-level factors associated with premature mortality. 1990 US Census data and mortality data from Chicago were merged with data from PHDCN, a study of 8782 residents in 343 Chicago neighborhoods. We performed a multivariate analysis to determine the association between premature mortality and concentrated disadvantage, residential stability, immigrant concentration, "collective efficacy" (a measure of willingness to help out for the common good), and "broken windows" (boarded up stores and homes, litter, and graffiti). Both collective efficacy and broken windows appeared to mediate the effect of concentrated disadvantage on all-cause premature mortality and mortality from cardiovascular disease and homicide, but there was also an interaction between broken windows and collective efficacy. Non-income characteristics associated with poverty should be further investigated. Interventions to determine whether these factors are causally related to health are needed.
\end{abstract}

(C) 2003 Elsevier Science Ltd. All rights reserved.

Keywords: Health disparities; Social and physical environment; Collective efficacy; USA

\section{Introduction}

Disparities in mortality rates by socioeconomic status (SES) are pronounced not just for all-cause mortality, but also for all major categories of specific causes of mortality, such as cardiovascular disease, cancer, and injuries (Davey-Smith, Neaton, Wentworth, Stamler, \& Stamler, 1996a; Davey-Smith, Wentworth, Neaton, Stamler, \& Stamler, 1996b; Kunst, Groenhof, \& Mackenbach, 1998; Mackenbach et al., 1999; Marmot, Shipley, \& Rose 1984; Yeracaris \& Kim, 1978). Rate ratios for mortality among persons in the lowest SES groups compared to those in the highest groups have been in the range of 1.2-4.0, indicating that the effect of SES on mortality is strong. These mortality differences

*Corresponding author. RAND Corporation, 1700 Main Street, 2138, Santa Monica, CA, USA. Tel.: + 1-310-393-0411; fax: + 1-310-393-4818.

E-mail address: dcohen@rand.org (D.A. Cohen). have been found in many different industrialized countries, including those with smaller ranges of socioeconomic difference than occur in the US and those with universal health care coverage (Mackenbach et al., 1999). The fact that these disparities cut across many different health processes, each of which has a distinct pathogenesis, suggests that there may be some underlying phenomenon related to SES that causes disease. It further suggests that our understanding of this phenomenon will come not from a closer study of individual diseases but rather from a study of issues outside of the individual disease pathways.

Some of the disparities in mortality associated with SES can be explained by lifestyle. For example, persons of lower SES are more likely to smoke, to drink to excess, and to have high-fat diets (Davey-Smith et al., 1996a, b; Marmot et al., 1991; Yeracaris \& Kim, 1978). Of note, there is a short-term economic cost of maintaining these risky behaviors, such as the cost of purchasing cigarettes, alcohol, or high-fat foods, which 
suggests that the unhealthier lifestyles are not due to an absolute lack of money. Why persons of lower SES have unhealthier lifestyles is both an important and an unanswered question.

Even more broadly, it is unclear why relative poverty, defined only in relation to the average resources available in a society and not necessarily with a lack of sufficient food, clothing, or shelter, is related to ill health (McCally et al., 1998). Researchers have investigated the possibility that SES-related health disparities are mediated by the number and nature of relationships among persons. At the individual level this concept has been measured as "social support" (the number and type of relationships an individual has) and at the population level this concept has been measured with the closely related concepts of "social organization" (Sampson, Raudenbush, \& Earls, 1997), "collective efficacy," a measure of willingness to help out for the common good (Sampson et al., 1997), and "social capital" (Kawachi \& Kennedy, 1997; Kennedy, Kawachi, Glass, \& Prothrow-Stith, 1998; Sampson et al., 1997), a measure of the quality of relationships among community residents. Communities with lower SES have lower rates of social cohesion (Coleman, 1988) and individuals in lower SES groups have less social support (Berkman \& Breslow, 1983). Researchers have consistently found that individuals with lower levels of social support have substantially higher mortality rates, with relative risks in the range of 1.8 4.0 (Kawachi et al., 1996; Sampson \& Laub, 1990; Welin, Larsson, Svardsudd, Tibblin, \& Tibblin, 1991). At the population level, communities with lower levels of social capital also have substantially higher mortality rates (Kawachi \& Kennedy, 1997). However, studies which have been able to look at the interrelationships among mortality, SES, and individual- or populationlevel social network measures suggest that differences in social cohesion cannot fully explain the disparities in mortality by SES (Berkman \& Syme, 1979; Haan, Kaplan \& Camacho, 1987; Kawachi et al., 1996; Kawachi \& Kennedy, 1997).

Another line of inquiry has been the study of the physical aspects of the neighborhoods in which persons of lower SES live. Neighborhoods of persons of lower SES tend to have higher rates of physical disorder and deterioration and also are characterized by social disorder (Sampson \& Groves, 1989; Shaw, David, Danny, Richard, \& Davey Smith, 2000). They also are more likely to have features that directly promote unhealthier lifestyles, such as a higher density of alcohol outlets (LaVeist \& Wallace Jr., 2000) and a lack of or higher prices for healthy foods at grocery stores (Macintyre, MacIver, \& Sooman, 1993). When it has been studied, the neighborhood of residence is an important predictor of mortality. In the largest US cohort study published demonstrating the deleterious effect of SES on mortality, the zip code of residence was used as a proxy for individual SES, so part or all of the observed effect may in fact have been an effect of neighborhood of residence, rather than an effect of individual SES (Davey-Smith et al., 1996a,b; House, Landis, \& Umberson, 1988; McCally et al., 1998). Haan et al. found that residents in a federally designated "poverty area" in Alameda County had a 1.7-fold higher mortality rate than residents in nonpoverty areas of the same county after controlling for demographic features, socioeconomic factors, lifestyle factors, and psychosocial factors (Haan et al., 1987). Cohen et al. (2000) found that after controlling for income, race, unemployment and education, deteriorated neighborhoods, defined by litter, graffiti, abandoned cars and blighted housing, were an independent predictor of gonorrhea rates. Indeed, there is increasing evidence that the physical environment is an important predictor of physical activity, a risk factor for a variety of diseases, including heart disease, diabetes, and certain types of cancer (Kahn et al., 2002; Sallis \& Owen, 1996).

In order to further explore the role of the social and physical environment on health we reanalyzed data from the Project on Human Development in Chicago Neighborhoods (PHDCN), a study that investigated collective efficacy in 343 neighborhood clusters (NC) in Chicago.

\section{Methods}

Data for this study were obtained from three separate sources. Individual deaths for Chicago residents from 1995 to 1998 were supplied by CAGIS, University of Illinois. These included all deaths registered in vital records by the Illinois Department of Public Health. The PHDCN provided data from a Community Survey (CS) completed in 1995 on 8782 residents of 343 neighborhoods in Chicago (Sampson et al., 1997). Included in the community questionnaire were information on neighborhood conditions, informal and formal social control, and social cohesion. Households within NC were sampled in such a way as to make a self-weighting sample. Finally, other predictor or control variables were obtained at the census tract level for Cook County (Chicago) from the 1990 US Census through their internet site.

All analyses were conducted at the neighborhood cluster level. NC were aggregations of census tracts grouped based on homogeneity of race, SES, housing density, and family organizations, with a mean of two census tracts per NC (range 1-7). The CS was an inhome interview and provided the link between census tracts and NC. Individual deaths and census tract data were aggregated to the NC level. 


\section{Mortality data}

Individual deaths were aggregated to the $\mathrm{NC}$ level and then age adjusted to calculate mortality rates (Page, Cole, \& Timmreck, 1995, Chap. 2). As the primary focus was on identifying what aspects of neighborhood conditions lead to poor health, premature deaths were selected as the most appropriate outcome measure. Deaths before $65 \mathrm{yr}$ of age from four consecutive years (1995-1998), were combined to stabilize the mortality estimates. Demographic data from the 1990 US Census by census tract were used in the adjustment. For adjustment, deaths for 1995-1998 were grouped into ten age groups as follows: less than 1 year, 1-4, 5-14, 15-24, 25-34, 35-54, 55-64 yr. In addition to total premature deaths, premature deaths by three specific causes were analyzed separately. These included: cardiovascular disease, homicide, and malignant neoplasms. These three causes comprise $54 \%$ of all premature deaths in the $343 \mathrm{NCs}$ (range from $29 \%$ to $74 \%$ ). All mortality rates were expressed as per 1000 population. All mortality rates except malignant neoplasms were log-transformed to reduce skewness. The log of homicide rate was adjusted by adding a constant to allow for zero values for some neighborhoods.

\section{Community survey predictors}

Data from the CS were analyzed similarly to the procedure used by Sampson et al. (1997). Collective efficacy was derived from two measures; "informal social control" (ISC) and "social cohesion and trust" (SCT). ISC measures the likelihood of intervention by neighbors when a problem arises. SCT includes questions about the neighbor's relationships with one another (i.e. trustworthiness, friendliness, helpfulness). Individual answers were aggregated to the NC by taking the mean of all pertinent questions and coded to measure from better to worse (i.e. a low value for collective efficacy reflects good relationships with the neighbors).

Measures of the physical environment of the neighborhood were also obtained from the CS. Respondents were asked to rank the following three items: (1) How much of a problem is litter, broken glass, or trash on the sidewalks and streets? (2) How much of a problem is graffiti on buildings and walls? and (3) How much of a problem are vacant or deserted houses or storefronts? The mean of these three questions was calculated for all NCs. The resulting measure was translated into an index, described in the next section.

\section{US census predictors}

All US census data from Cook County (Chicago) were extracted from the US Census Bureau internet site; however, only those corresponding to the NCs used in the CS were included. The following variables were selected: the number of females, number of blacks, number living in poverty, number receiving public assistance, female headed-households, number unemployed, less than $18 \mathrm{yr}$ of age, number of Hispanics, foreign born, who lived in the same house $5 \mathrm{yr}$ previously, of owner occupied houses, of total units boarded-up. All estimates were aggregated to the $\mathrm{NC}$ level and percentages calculated using appropriate denominators.

The housing measure of number of total units boarded-up was aggregated to the $\mathrm{NC}$ level and expressed as a percent of all units. It was combined with measures of the physical environment from the CS. The resulting "broken windows" index was the mean of the normalized estimates for both the CS and the census tract variables. All components of the index were coded to go from better to worse; thus, a low value for broken windows reflects lower neighborhood deterioration.

\section{Data analysis}

We used the same factors that were found to predict violence in the original study of collective efficacy (Sampson et al., 1997): concentrated disadvantage, immigrant concentration, and residential stability. The factor analysis procedure in SPSS gave factor loadings, extracted using the oblique method. Least-squares regression analysis was used to examine relationships of the three factors, collective efficacy, and the physical environment (broken windows) on mortality. Although Sampson used hierarchical linear modeling to examine neighborhood affects on violence after controlling for selected individual measures, this could not be duplicated here because mortality data came from a different source than the individual data on collective efficacy.

Casewise diagnostics and collinearity statistics were calculated to identify any outlying values and problems with correlations between predictors. Percent female was added as a control variable since gender is related to premature death, and these rates were only adjusted for age. The broken windows index was added to the model along with collective efficacy to determine whether the physical environment had any independent effect on premature mortality. We also included an interaction term between collective efficacy and broken windows. We used the logistic regression procedure in SPSS to calculate odds ratios (OR) for the effect of the collective efficacy and broken windows indices on premature mortality, controlling for the other predictors, concentrated disadvantage, residential stability, immigrant concentration, female headed household, percent female and the interaction between broken windows and collective efficacy. Age-adjusted premature mortality, and the indices were coded into 0 or 1 as follows: one or 
Table 1

Descriptive statistics for all outcome and predictor variables ${ }^{\mathrm{a}}$

\begin{tabular}{|c|c|c|c|c|}
\hline Variable & Mean & SD & Minimum & Maximum \\
\hline Age adjusted total premature death rate & 17.53 & 7.69 & 2.93 & 47.28 \\
\hline Age adjusted premature death rate due to cardiovascular disease & 4.68 & 2.34 & 0.59 & 15.19 \\
\hline Age adjusted premature death rate due to homicide & 1.23 & 1.07 & 0 & 6.78 \\
\hline Age adjusted premature death rate due to malignant neoplasms & 3.50 & 1.41 & 0.51 & 7.42 \\
\hline Percent female & 52.31 & 2.94 & 43.97 & 62.70 \\
\hline Collective efficacy ${ }^{\mathrm{b}}$ (low is good) & 2.59 & 0.33 & 1.52 & 3.43 \\
\hline Broken windows index ${ }^{c}$ (low is good) & 0 & 0.90 & -1.75 & 2.49 \\
\hline $\mathrm{CD}^{\mathrm{d}}$ - percent living in poverty & 24.69 & 19.15 & 0.79 & 99.06 \\
\hline $\mathrm{CD}$ - percent households on public assistance & 17.53 & 15.07 & 1.03 & 76.61 \\
\hline $\mathrm{CD}$ - percent female headed families & 32.98 & 18.58 & 6.92 & 94.73 \\
\hline $\mathrm{CD}$-percent unemployed & 13.94 & 9.65 & 1.93 & 54.19 \\
\hline $\mathrm{CD}$ - percent population $<18 \mathrm{yr}$ old & 27.17 & 9.09 & 4.04 & 55.47 \\
\hline $\mathrm{CD}$ - percent black & 41.42 & 43.75 & 0 & 100 \\
\hline $\mathrm{IC}$ - percent foreign born & 16.59 & 15.70 & 0 & 65.27 \\
\hline $\mathrm{IC}$ - percent Hispanic & 19.95 & 26.01 & 0 & 96.26 \\
\hline $\mathrm{RS}$ - lived in same area past $5 \mathrm{yr}$ & 51.56 & 11.84 & 24.60 & 78.10 \\
\hline RS - percent units owner occupied & 42.76 & 23.38 & 0 & 93.44 \\
\hline
\end{tabular}

${ }^{\text {a }}$ Sample is 343 neighborhoods. All mortality rates are per 1000 population.

${ }^{\mathrm{b}} 1$. Scaled such that a lower index reflects greater collective efficacy.

${ }^{c} 2$. Scaled such that a lower index reflects less neighborhood deterioration.

${ }^{\mathrm{d}} \mathrm{CD}=$ concentrated disadvantage factor; $\mathrm{IC}=$ immigrant concentration factor; $\mathrm{RS}=$ residential stability factor.

more standard deviation above the mean was coded disease present, and exposure (yes) and less than $1 \mathrm{SD}$ above the mean was disease absent, and exposure (no).

\section{Results}

Descriptive statistics for the $343 \mathrm{NC}$ are listed in Table 1. There was good variability of all measures across the 343 neighborhoods. Table 2 shows correlations between all the variables. Of note are the very high bivariate correlations between concentrated disadvantage and all the other variables, especially the broken windows index $(r=0.85)$, premature mortality $(r=0.80)$, and collective efficacy $(r=0.60)$. Casewise diagnostics and collinearity statistics indicated that the high correlations were not problematic, in that both the variance inflation factors and condition indices were below 5.0, considering that only levels $>10$ and $>30$, respectively, are considered unacceptable (Kleinbaum, Kupper, \& Muller, 1998).

Table 3 shows four different regression models with premature mortality and cause-specific premature mortality as the dependent variable. In the first model, allcause premature mortality was associated with all the factors except residential stability. The addition of collective efficacy only slightly improved the variance explained, but significantly reduced the contribution of concentrated disadvantage to premature mortality. The addition of the broken windows index further reduced the contribution of concentrated disadvantage. Thus, both collective efficacy and concentrated disadvantage met the definition of a mediator (Baron \& Kenny, 1986). There was a significant interaction between broken windows and collective efficacy $(\beta=-0.244, p=0.03)$ and the addition of this interaction term significantly increased the strength of the independent associations between both collective efficacy and broken windows and premature mortality. In neighborhoods with low broken windows scores, collective efficacy appeared to be protective for premature mortality, while in neighborhoods with high broken windows scores, there was no effect of collective efficacy on premature mortality $(p=0.45)$.

For premature mortality due to cardiovascular disease and homicide, similar patterns emerged: adding the collective efficacy and broken windows indices reduced the contribution of concentrated disadvantage. For both cardiovascular disease and homicide, adding the interaction term significantly improved the relationship between mortality and broken windows and collective efficacy. Neither collective efficacy nor broken windows were associated with premature mortality from malignant neoplasms.

In the logistic regression model predicting premature mortality, the odds ratio for broken windows was 6.99 $(p=0.05)$, indicating that in the most deteriorated neighborhoods the odds of dying prematurely was increased nearly 7-fold. After controlling for the interaction with broken windows, the odds ratio for 
Table 2

Correlations among all outcome and predictor variables

\begin{tabular}{|c|c|c|c|c|c|c|c|c|c|c|c|}
\hline & & $\begin{array}{l}\text { Log of total } \\
\text { premature } \\
\text { morality rate } \\
1995-1998\end{array}$ & $\begin{array}{l}\text { Log of } \\
\text { premature } \\
\text { mortality rate } \\
\text { from } \\
\text { cardiovascular } \\
\text { disease } 1995- \\
1998\end{array}$ & $\begin{array}{l}\text { Log of } \\
\text { premature } \\
\text { mortality rate } \\
\text { from homicide } \\
1995-1998\end{array}$ & $\begin{array}{l}\text { Premature age } \\
\text { adjusted death } \\
\text { rate from } \\
\text { malignant } \\
\text { neoplasms } \\
\text { 1995-1998 }\end{array}$ & $\begin{array}{l}\text { Factor 1: } \\
\text { Sampson's } \\
\text { concentrated } \\
\text { disavantage }\end{array}$ & $\begin{array}{l}\text { Factor 2: } \\
\text { Sampson's } \\
\text { immigrant } \\
\text { concentration }\end{array}$ & $\begin{array}{l}\text { Factor 3: } \\
\text { Sampson's } \\
\text { residential } \\
\text { stability }\end{array}$ & $\begin{array}{l}\% \text { female } \\
\text { (centered, less } \\
\text { mean) }\end{array}$ & $\begin{array}{l}\text { Collective } \\
\text { efficacy-low is } \\
\text { good }\end{array}$ & $\begin{array}{l}\text { Broken } \\
\text { windows index } \\
\text { (mean of } \\
\text { zlogvbrd \& } \\
\text { zbwinkx) }\end{array}$ \\
\hline \multirow{2}{*}{$\begin{array}{l}\text { Log of total } \\
\text { premature } \\
\text { morality rate } \\
\text { 1995-1998 }\end{array}$} & Pearson $R$ & 1.000 & $* *$ & $* *$ & $* *$ & $* *$ & $* *$ & & $* *$ & $* *$ & $* *$ \\
\hline & $\begin{array}{l}\text { Signif. }(p) \\
N\end{array}$ & $\overline{344}$ & & & & & & & & & \\
\hline \multirow{2}{*}{$\begin{array}{l}\text { Log of } \\
\text { premature } \\
\text { mortality rate } \\
\text { from } \\
\text { cardiovascular } \\
\text { disease } 1995- \\
1998\end{array}$} & Pearson $R$ & $0.917^{\text {*** }}$ & 1.000 & $* *$ & $* *$ & $* *$ & $* *$ & & $* *$ & $* * *$ & $* *$ \\
\hline & Signif. ( $p$ ) & 0.000 & - & & & & & & & & \\
\hline \multirow{3}{*}{$\begin{array}{l}\text { Log of } \\
\text { premature } \\
\text { mortality rate } \\
\text { from homicide } \\
\text { 1995-1998 }\end{array}$} & Pearson $R$ & $0.765^{* * *}$ & $0.667^{* * *}$ & 1.000 & $* *$ & $* *$ & $* *$ & $* *$ & & $* * *$ & $* * *$ \\
\hline & Signif. $(p)$ & 0.000 & 0.000 & - & & & & & & & \\
\hline & $N$ & 321 & 321 & 321 & & & & & & & \\
\hline \multirow{3}{*}{$\begin{array}{l}\text { Premature age } \\
\text { adjusted death } \\
\text { rate from } \\
\text { malignant } \\
\text { neoplasms } \\
\text { 1995-1998 }\end{array}$} & Pearson $R$ & $0.761^{* * *}$ & $0.660^{* * * *}$ & $0.515^{* * *}$ & 1.000 & $* *$ & $* *$ & & $* *$ & $* * *$ & $* * *$ \\
\hline & Signif. $(p)$ & 0.000 & 0.000 & 0.000 & - & & & & & & \\
\hline & $N$ & 344 & 344 & 321 & 344 & & & & & & \\
\hline \multirow{2}{*}{$\begin{array}{l}\text { Factor 1: } \\
\text { Sampson's } \\
\text { concentrated } \\
\text { disavantage }\end{array}$} & Pearson $R$ & $0.802^{* * *}$ & $0.724^{* * *}$ & $0.740^{\text {** }}$ & $563^{* *}$ & 1.000 & $* *$ & & $* *$ & $* *$ & $* *$ \\
\hline & Signif. $(p)$ & 0.000 & 0.000 & 0.000 & 0.000 & - & & & & & \\
\hline
\end{tabular}




\begin{tabular}{|c|c|c|c|c|c|c|c|c|c|c|c|}
\hline & & $\begin{array}{l}\text { Log of total } \\
\text { premature } \\
\text { morality rate } \\
\text { 1995-1998 }\end{array}$ & $\begin{array}{l}\text { Log of } \\
\text { premature } \\
\text { mortality rate } \\
\text { from } \\
\text { cardiovascular } \\
\text { disease 1995- } \\
1998\end{array}$ & $\begin{array}{l}\text { Log of } \\
\text { premature } \\
\text { mortality rate } \\
\text { from homicide } \\
\text { 1995-1998 }\end{array}$ & $\begin{array}{l}\text { Premature age } \\
\text { adjusted death } \\
\text { rate from } \\
\text { malignant } \\
\text { neoplasms } \\
\text { 1995-1998 }\end{array}$ & $\begin{array}{l}\text { Factor 1: } \\
\text { Sampson's } \\
\text { concentrated } \\
\text { disavantage }\end{array}$ & $\begin{array}{l}\text { Factor 2: } \\
\text { Sampson's } \\
\text { immigrant } \\
\text { concentration }\end{array}$ & $\begin{array}{l}\text { Factor 3: } \\
\text { Sampson's } \\
\text { residential } \\
\text { stability }\end{array}$ & $\begin{array}{l}\% \text { female } \\
\text { (centered, less } \\
\text { mean) }\end{array}$ & $\begin{array}{l}\text { Collective } \\
\text { efficacy-low is } \\
\text { good }\end{array}$ & $\begin{array}{l}\text { Broken } \\
\text { windows index } \\
\text { (mean of } \\
\text { zlogvbrd \& } \\
\text { zbwinkx) }\end{array}$ \\
\hline & $N$ & 343 & 343 & 320 & 343 & 343 & & & & & \\
\hline \multirow{3}{*}{$\begin{array}{l}\text { Factor 2: } \\
\text { Sampson's } \\
\text { immigrant } \\
\text { concentration }\end{array}$} & Pearson $R$ & $-0.435^{* *}$ & $-0.448^{* * *}$ & $-0.292^{* *}$ & $-0.549^{* *}$ & $-0.182^{* *}$ & 1.000 & $* *$ & $* *$ & & \\
\hline & Signif. $(p)$ & 0.000 & 0.000 & 0.000 & 0.000 & 0.001 & - & & & & \\
\hline & $N$ & 343 & 343 & 320 & 343 & 343 & 343 & & & & \\
\hline \multirow{3}{*}{$\begin{array}{l}\text { Factor 3: } \\
\text { Sampson's } \\
\text { residential } \\
\text { stability }\end{array}$} & Pearson $R$ & -0.52 & -0.25 & 0.106 & $0.090^{* *}$ & -0.076 & $-0.183^{* *}$ & 1.000 & $* *$ & *** & $* *$ \\
\hline & Signif. $(p)$ & 0.337 & 0.644 & 0.058 & 0.096 & 0.159 & 0.001 & - & & & \\
\hline & $N$ & 343 & 343 & 320 & 343 & 343 & 343 & 343 & & & \\
\hline \multirow{3}{*}{$\begin{array}{l}\% \text { Female } \\
\text { (centered, less } \\
\text { mean) }\end{array}$} & Pearson $R$ & $0.451^{* * *}$ & $0.445^{* *}$ & $0.355^{* * *}$ & $0.510^{* *}$ & $0.405^{* *}$ & -0.692 & $0.233^{* *}$ & 1.000 & & \\
\hline & Signif. $(p)$ & 0.000 & 0.000 & 0.000 & 0.000 & 0.000 & 0.000 & 0.000 & - & & \\
\hline & $N$ & 343 & 343 & 320 & 343 & 343 & 343 & 343 & 343 & & \\
\hline \multirow{3}{*}{$\begin{array}{l}\text { Collective } \\
\text { efficacy-low is } \\
\text { good }\end{array}$} & Pearson $R$ & $0.535^{* * *}$ & $0.462^{* * *}$ & $0.436^{* * *}$ & $0.240^{* *}$ & $0.602^{* *}$ & 0.079 & $-0.435^{* *}$ & $0.023^{* *}$ & 1.000 & \\
\hline & Signif. $(p)$ & 0.000 & 0.000 & 0.000 & 0.000 & 0.000 & 0.146 & 0.000 & 0.675 & - & \\
\hline & $N$ & 343 & 343 & 320 & 343 & 343 & 343 & 343 & 343 & 343 & \\
\hline \multirow{3}{*}{$\begin{array}{l}\text { Broken } \\
\text { windows index }\end{array}$} & Pearson $R$ & $0.700^{* * *}$ & $0.633^{* * *}$ & $0.666^{* * *}$ & $409^{* *}$ & $0.847^{* *}$ & -0.006 & $-0.181^{* *}$ & $0.142^{* *}$ & $0.698^{* *}$ & 1.000 \\
\hline & Signif. $(p)$ & 0.000 & 0.000 & 0.000 & 0.000 & 0.000 & 0.911 & 0.001 & 0.008 & 0.000 & - \\
\hline & $N$ & 343 & 343 & 320 & 343 & 343 & 343 & 343 & 343 & 343 & 343 \\
\hline
\end{tabular}

** Correlation is significant at the 0.01 level (two-tailed). 
Table 3

Results of regression analysis using premature mortality as dependent variable

\begin{tabular}{|c|c|c|c|c|c|c|c|c|c|}
\hline & & \multicolumn{4}{|c|}{ Total premature mortality rate ${ }^{a}$} & \multicolumn{4}{|c|}{ Cardiovascular mortality rate } \\
\hline & & $\mathrm{w} / \mathrm{o} \mathrm{CE} \mathrm{CE}^{3}$ & $\mathrm{w} / \mathrm{CE}$ & $\mathrm{w} / \mathrm{BW}$ & $\mathrm{w} / \mathrm{Int}$ & w/o CE & $\mathrm{w} / \mathrm{CE}$ & $\mathrm{w} / \mathrm{BW}$ & $\mathrm{w} / \mathrm{Int}$ \\
\hline \multirow{3}{*}{$\begin{array}{l}\text { Concentrated } \\
\text { disadvantage }\end{array}$} & Beta & 0.782 & 0.682 & 0.617 & 0.603 & 0.715 & 0.633 & 0.544 & 0.487 \\
\hline & $t$ & 26.010 & 17.978 & 12.506 & 10.545 & 20.011 & 13.882 & 9.181 & 6.992 \\
\hline & $p$-value & 0.000 & 0.000 & 0.000 & 0.000 & 0.000 & 0.000 & 0.000 & 0.000 \\
\hline \multirow{3}{*}{$\begin{array}{l}\text { Immigrant } \\
\text { concentration }\end{array}$} & Beta & -0.417 & -0.423 & -0.408 & -0.420 & -0.415 & -0.419 & -0.401 & -0.416 \\
\hline & $t$ & -11.102 & -11.518 & -10.952 & -11.901 & -9.509 & -9.707 & -9.174 & -9.874 \\
\hline & $p$-value & 0.000 & 0.000 & 0.000 & 0.000 & 0.000 & 0.000 & 0.000 & 0.000 \\
\hline \multirow[t]{3}{*}{ Residential stability } & Beta & -0.050 & 0.006 & -0.005 & 0.018 & -0.029 & 0.017 & 0.001 & 0.022 \\
\hline & $t$ & -1.795 & 0.194 & -0.163 & 0.614 & -0.868 & 0.458 & 0.040 & 0.615 \\
\hline & $p$-value & 0.074 & 0.846 & 0.871 & 0.539 & 0.386 & 0.648 & 0.968 & 0.539 \\
\hline \multirow[t]{3}{*}{ Percent female } & Beta & -0.141 & -0.121 & -0.105 & -0.079 & -0.125 & -0.108 & -0.085 & -0.053 \\
\hline & $t$ & -3.428 & -2.992 & -2.565 & -1.939 & -2.590 & -2.236 & -1.752 & -1.083 \\
\hline & $p$-value & 0.001 & 0.003 & 0.011 & 0.053 & 0.010 & 0.026 & 0.081 & 0.280 \\
\hline \multirow[t]{3}{*}{ Collective efficacy } & Beta & & 0.158 & 0.145 & 0.364 & & 0.129 & 0.112 & 0.260 \\
\hline & $t$ & - & 4.125 & 3.764 & 5.478 & - & 2.840 & 2.449 & 3.250 \\
\hline & $p$-value & & 0.000 & 0.000 & 0.000 & & 0.005 & 0.015 & 0.001 \\
\hline \multirow[t]{3}{*}{ Broken windows } & Beta & & & 0.088 & 0.520 & & & 0.119 & 0.504 \\
\hline & $t$ & - & - & 2.053 & 5.389 & - & - & 2.343 & 4.339 \\
\hline & $p$-value & & & 0.041 & 0.000 & & & 0.020 & 0.000 \\
\hline \multirow{3}{*}{$\begin{array}{l}\text { Interaction of } \\
\text { broken windows and } \\
\text { collective efficacy }\end{array}$} & Beta & & & & -0.605 & & & & -0.461 \\
\hline & $t$ & - & - & - & -4.596 & - & - & - & -2.905 \\
\hline & $p$-value & & & & 0.000 & & & & 0.004 \\
\hline \multirow{2}{*}{\multicolumn{2}{|c|}{$\begin{array}{l}N \\
\text { Adjusted } R^{2}\end{array}$}} & 342 & & & & 338 & & & \\
\hline & & 0.757 & 0.768 & 0.772 & 0.785 & 0.667 & 0.674 & 0.679 & 0.691 \\
\hline
\end{tabular}




\begin{tabular}{|c|c|c|c|c|c|c|c|c|c|}
\hline & & \multicolumn{4}{|c|}{ Homicide rate } & \multicolumn{4}{|c|}{ Malignant neoplasm mortality rate } \\
\hline & & w/o CE & $\mathrm{w} / \mathrm{CE}$ & $\mathrm{w} / \mathrm{BW}$ & $\mathrm{w} / \mathrm{Int}$ & w/o CE & $\mathrm{w} / \mathrm{CE}$ & $\mathrm{w} / \mathrm{BW}$ & $\mathrm{w} / \mathrm{Int}$ \\
\hline \multirow{3}{*}{$\begin{array}{l}\text { Concentrated } \\
\text { disadvantage }\end{array}$} & Beta & 0.804 & 0.664 & 0.605 & 0.599 & 0.499 & 0.485 & 0.479 & 0.489 \\
\hline & $t$ & 22.663 & 14.947 & 10.452 & 8.805 & 12.057 & 9.033 & 6.800 & 5.821 \\
\hline & $p$-value & 0.000 & 0.000 & 0.000 & 0.000 & 0.000 & 0.000 & 0.000 & 0.000 \\
\hline \multirow{3}{*}{$\begin{array}{l}\text { Immigrant } \\
\text { concentration }\end{array}$} & Beta & -0.205 & -0.216 & -0.202 & -0.214 & -0.459 & -0.460 & -0.459 & -0.460 \\
\hline & $t$ & -4.657 & -5.056 & -4.656 & -5.131 & -8.860 & -8.860 & -8.619 & -8.857 \\
\hline & $p$-value & 0.000 & 0.000 & 0.000 & 0.000 & 0.000 & 0.000 & 0.000 & 0.000 \\
\hline \multirow[t]{3}{*}{ Residential stability } & Beta & 0.107 & 0.184 & 0.174 & 0.195 & 0.056 & 0.064 & 0.063 & 0.072 \\
\hline & $t$ & 3.237 & 5.186 & 4.850 & 5.574 & 1.459 & 1.493 & 1.444 & 1.644 \\
\hline & $p$-value & 0.001 & 0.000 & 0.000 & 0.000 & 0.146 & 0.136 & 0.150 & 0.101 \\
\hline \multirow[t]{3}{*}{ Percent female } & Beta & -0.101 & -0.075 & -0.060 & -0.038 & -0.005 & -0.003 & -0.001 & 0.007 \\
\hline & $t$ & -2.082 & -1.580 & -1.255 & -0.783 & -0.094 & -0.048 & -0.019 & 0.109 \\
\hline & $p$-value & 0.038 & 0.115 & 0.210 & 0.434 & 0.925 & 0.962 & 0.985 & 0.913 \\
\hline \multirow[t]{3}{*}{ Collective efficacy } & Beta & & 0.221 & 0.210 & 0.416 & & 0.023 & 0.022 & 0.133 \\
\hline & & - & 4.956 & 4.655 & 5.294 & - & 0.424 & 0.396 & 1.370 \\
\hline & $p$-value & & 0.000 & 0.000 & 0.000 & & 0.672 & 0.692 & 0.172 \\
\hline \multirow[t]{3}{*}{ Broken windows } & Beta & & & 0.079 & 0.473 & & & 0.008 & 0.190 \\
\hline & & - & - & 1.577 & 4.131 & - & - & 0.132 & 1.343 \\
\hline & $p$-value & & & 0.116 & 0.000 & & & 0.895 & 0.180 \\
\hline \multirow{3}{*}{$\begin{array}{l}\text { Interaction of } \\
\text { broken windows and } \\
\text { collective efficacy }\end{array}$} & Beta & & & & -0.564 & & & & -0.284 \\
\hline & $t$ & - & - & - & -3.617 & - & - & - & -1.475 \\
\hline & $p$-value & & & & 0.000 & & & & 0.141 \\
\hline$N$ & & 343 & & & & 341 & & & \\
\hline Adjusted $R^{2}$ & & 0.661 & 0.683 & 0.685 & 0.697 & 0.540 & 0.539 & 0.538 & 0.539 \\
\hline
\end{tabular}


the effect of collective efficacy on premature mortality was $0.28(p=0.19)$.

\section{Discussion}

The constructs of collective efficacy and broken windows are likely to be two mechanisms through which relative poverty leads to poor health. Collective efficacy is not only likely to constrain behaviors associated with homicide, but it likely to be related to the concepts of social support that have been found to be associated with increased longevity in other studies (Berkman, Glass, Brissette, \& Seeman, 2000). While the mechanism of how social interactions influence physiological properties in adults is unknown, one clinical trial demonstrated that persons with more social ties were less susceptible to development of cold symptoms after receiving nasal drops of rhinovirus suggesting a role of the immune system (Cohen, Doyle, Skoner, Rabin, \& Gwaltney, 1997). A randomized controlled clinical trial to increase social interactions with infants in intensive care units has shown significant differences in medical outcomes, including improved recovery rates, shorter hospital stays, better weight gain, better motor system functioning and autonomic regulation among infants who received more human attention compared to the usual standard of care (Als et al., 1994). There is no doubt that social interactions influence physiological functioning in infancy; therefore, it is plausible that social interactions can have physiological effects on adults as well.

The broken windows construct, which signifies deteriorated neighborhoods, is likely to be associated with decreased physical activity, an important contributor to cardiovascular mortality. Recent studies on "walkability" and environmental determinants of physical activity have indicated that attractive, aesthetically pleasing settings are more conducive to physical activity than areas that are unattractive and possibly dangerous (Brownson et al., 2000; King et al., 2000). The presence of a blighted house in an older urban neighborhood may discourage walking by inducing fear in residents - fear that may be irrational (based on the message communicated by the appearance of the house) or rational (based on the abandoned house becoming a site for prostitution, drug sales, and associated violence). This effect, though indirect, may be very strong; in one survey $31 \%$ of low-income persons are afraid to walk or jog in their neighborhoods, compared to $15 \%$ of higherincome persons (Yankelovich, 1995). While the dominant thinking about health is that individual knowledge, attitudes and skills determine our health behaviors, the possibility that the conditions in which we live are in large part responsible for longevity suggests that future public health interventions should consider our social and physical environments.

The construct of broken windows, should it literally have an effect on healthy behaviors, is potentially remediable through enforcement of existing zoning and housing codes. All localities have agencies that govern the maintenance of public and private poverty. Cities can make special efforts to keep streets clear and clean, and to swiftly adjudicate abandoned properties, including potential expropriation and transformation of blighted buildings to green spaces or small parks. Currently, authorities tend to be more lax in enforcing housing codes in lower-income areas than in higherincome areas (Howe, 1983; Ross, 1995). Indeed, the maintenance of public areas may be more important in low-income neighborhoods than in high-income neighborhoods.

The construct of collective efficacy is also potentially remediable, although the mechanisms for such remediation are not clear. Participation in voluntary organizations may be a means to develop trust and better social relationships, rather than participation being only a reflection of already existing positive social skills (Brehm \& Rahn, 1997). If this is true, than the development and support of a variety of local civic organizations and clubs may provide opportunities for more positive social interactions and social support among neighbors.

A strong relationship between the physical condition of one's environment and one's ability to develop supportive relationships with one's neighbors is certainly plausible. When neighbors fail to maintain their properties, it may be perceived as an assault on other residents who may see their own property values threatened. Neighbors may also be concerned about hazards to their personal well-being. When such threatening conditions continue without remediation or intervention by a governing authority, residents may feel more distrustful and powerless and unable (or unwilling) to develop positive social relationships. However, where the neighborhood environment is pleasant (few broken windows), people may be more likely to spend time outdoors, which would increase the likelihood that they would see and/or meet their neighbors and gain some familiarity with them or even have positive social interactions.

In general, both collective efficacy and broken windows show independent influences on premature mortality; however, the lack of effect of an independent effect of collective efficacy in deteriorated neighborhoods suggests that it may not be a viable leverage point for reducing premature mortality in these conditions. The physical stigma of poverty and the implied tolerance of deviant behaviors in areas marked by graffiti and boarded up homes may overwhelm the ability of people to act cooperatively for the greater good. The physical environment may act as a threshold such that beyond a certain point of deterioration, it may not be possible to 
actually initiate viable or sustainable voluntary organizations where there are no appropriate physical infrastructures to house them nor complementary structures that could provide additional support.

There are several limitations to this analysis. First, cross-sectional data cannot address the directionality of the association between the social and physical environments and mortality. On the one hand, it is possible that poorly kept neighborhoods lead to poor collective behavior, but it is also plausible that people with no interest in the common good are the cause of neighborhood deterioration. Second, many of the risk factors for premature mortality are cumulative and may have occurred when people lived elsewhere - only half of the sample reported living in the same household over the past 5 years. Third, this analysis is ecological and therefore unable to control for individual level factors such as health risk behaviors, particularly smoking, alcohol use or exercise. However, the findings are consistent with other studies that show relationships between social variables and mortality even after controlling for individual level factors. Furthermore, the association of physical environmental variables with mortality has a plausible physiological mechanism through physical activity. Finally, there is a time gap between the measures of neighborhood as reported by the US census in 1990 and the survey measures that were collected in 1995, during which neighborhood characteristics may have changed.

In order to determine causality, the next step would be to either evaluate natural experiments or to implement interventions to improve collective efficacy or reduce broken windows and look at relevant intermediate endpoints such as physical activity or measures of social interactions, such as membership in voluntary organizations. Given the likelihood that social and physical environments are in large part responsible for the health disparities that plague our nation, further efforts to identify the factors that are both causal and remediable are needed to improve population-level health.

\section{Acknowledgements}

We wish to acknowledge the assistance of Robert Sampson, Ph.D., University of Chicago and Felton Earls, MD, Harvard University for providing data from the Project of Human Development in Chicago Neighborhoods for their assistance in analysis and for their insightful comments on the manuscript.

\section{Appendix A}

Factor loadings are shown in Table 4.
Table 4

Factor loadings

\begin{tabular}{lll}
\hline Factor & Variables & $\begin{array}{l}\text { Factor } \\
\text { loadings }\end{array}$ \\
\hline $\begin{array}{l}\text { Concentrated } \\
\text { disadvantage }\end{array}$ & $\begin{array}{l}\text { Percent living in } \\
\text { poverty } \\
\text { Percent households } \\
\text { on public assistance }\end{array}$ & 0.926 \\
& $\begin{array}{l}\text { Percent female } \\
\text { headed households }\end{array}$ & 0.939 \\
& $\begin{array}{l}\text { Percent unemployed } \\
\text { Percent population } \\
\text { less than 18 yr }\end{array}$ & 0.939 \\
& $\begin{array}{l}\text { Percent black } \\
\text { Percent hispanic }\end{array}$ & 0.938 \\
Immigrant & 0.644 \\
concentration & $\begin{array}{l}\text { Percent foreign born } \\
\text { Percent stayed in } \\
\text { Residential }\end{array}$ & 0.723 \\
stability & $\begin{array}{l}\text { Pame area past 5 yr } \\
\text { occupied }\end{array}$ & 0.908 \\
& & 0.830 \\
\hline
\end{tabular}

\section{References}

Als, H., Lawhon, G., Duffy, F. H., McAnulty, G. B., GibesGrossman, R., \& Blickman, J. G. (1994). Individualized developmental care for the very low-birth-weight preterm infant. Medical and neurofunctional effects. Journal of the American Medical Association, 272, 853-858 (see comments).

Baron, R. M., \& Kenny, D. A. (1986). The moderator-mediator variable distinction in social psychological research: Conceptual, strategic, and statistical considerations. Journal of Personality and Social Psychology, 51, 117-1182.

Berkman, L. F., \& Breslow, L. (1983). Health and ways of living: The Alameda county study. New York: Oxford University Press.

Berkman, L. F., Glass, T., Brissette, I., \& Seeman, T. E. (2000). From social integration to health: Durkheim in the new millennium. Social Science \& Medicine, 51, 843-857.

Berkman, L. F., \& Syme, S. L. (1979). Social Networks, Host Resistance, and Mortality: A nine-year follow-up study of Alameda county residents. American Journal of Epidemiology, 190, 186-204.

Brehm, J., \& Rahn, W. (1997). Individual-level evidence for the causes and consequences of social capital. American Journal of Political Science, 41, 999-1023.

Brownson, R. C., Eyler, A. A., King, A. C., Brown, D. R., Shyu, Y. L., \& Sallis, J. F. (2000). Patterns and correlates of physical activity among US women 40 years and older. American Journal of Public Health, 90, 264-270.

Cohen, D. A., Spear, S., Scribner, R. A., Kissinger, P., Mason, K., \& Wildgen, J. (2000). Broken windows and the risk of gonorrhea. American Journal of Public Health, 90, 230-236.

Cohen, S., Doyle, W. J., Skoner, D. P., Rabin, B. S., \& Gwaltney, J. M. (1997). Social ties and susceptibility to the 
common cold. Journal of the American Medical Association, 277, 1940-1944 (see comments).

Coleman, J. S. (1988). Social capital in the creation of human capital. American Journal of Sociology, 94, S95-S120.

Davey-Smith, G., Neaton, J. D., Wentworth, D., Stamler, R., \& Stamler, J. (1996a). Socioeconomic differentials in mortality risk among men screened for the multiple risk factor intervention trial: I. White men. American Journal of Public Health, 86, 486-496.

Davey-Smith, G., Wentworth, D., Neaton, J. D., Stamler, R., \& Stamler, J. (1996b). Socioeconomic differentials in mortality risk among men screened for the multiple risk factor intervention trial: II. Black men. American Journal of Public Health, 86, 497-504.

Haan, M., Kaplan, G. A., \& Camacho, T. (1987). Poverty and health: Prospective evidence from the Alameda county study. American Journal of Epidemiology, 125, 989-998.

House, J. S., Landis, K. R., \& Umberson, D. (1988). Social relationships and health. Science, $84,541-545$.

Howe, E. (1983). Housing code enforcement in eleven cities. University of Detroit Journal of Urban Law, 60, 373-391.

Kahn, E. B., Ramsey, L. T., Brownson, R. C., Heath, G. W., Howze, E. H., Powell, K. E., Stone, E. J., Rajab, M. W., \& Corso, P. (2002). The effectiveness of interventions to increase physical activity. A systematic review (12). American Jounal of Preventive Medicine, 22, 73-107.

Kawachi, I., Colditz, G. A., Ascherio, A., Rimm, E. B., Giovannucci, E., Stampfer, M. J., \& Willette, W. C. (1996). A prospective study of social networks in relation to total mortality and cardiovascular disease in the USA. Journal of the Epidemiology and Community Health, 50, 245-251.

Kawachi, I., \& Kennedy, B. P. (1997). Socioeconomic determinants of health: Health and social cohesion: Why care about income inequality? British Medical Journal, 314, 1037-1040.

Kennedy, B. P., Kawachi, I., Glass, R., \& Prothrow-Stith, D. (1998). Income distribution, socioeconomic status, and self rated health in the United States: Multilevel analysis. British Medical Journal, 317, 917-921.

King, A. C., Castro, C., Wilcox, S., Eyler, A. A., Sallis, J. F., \& Brownson, R. C. (2000). Personal and environmental factors associated with physical inactivity among different racial-ethnic groups of US middle-aged and older-aged women. Health Psychology, 19, 354-364.

Kleinbaum, D. G., Kupper, L. L., \& Muller, K. E. (1998). Applied regression analysis and other multivariable methods. Boston: PWS-Kent Publishing.

Kunst, A. E., Groenhof, G., \& Mackenbach, J. P. (1998). Occupational class and cause-specific mortality in middleaged men in 11 European countries: Comparison of population based studies. British Medical Journal, 316, 1636-1642.
LaVeist, T. A., \& Wallace Jr., J. M. (2000). Health risk and inequitable distribution of liquor stores in African American neighborhood. Social Science \& Medicine, 51, 613-617.

Macintyre, S., MacIver, S., \& Sooman, A. (1993). Area, class and health: Should we be focusing on places or people? Journal of Social Policy, 22, 213-234.

Mackenbach, J. P., Kunst, A. E., Groenhof, F., Borgan, J-K., Cost, G., Faggiono, F., Jozan, P., Leinsalu, M., Martikainen, P., Rychtarikova, J., \& Vaikonen, T. (1999). Socioeconomic inequalities in mortality among men and women: An international study. American Journal of Public Health, 89, 1800-1806.

Marmot, M. G., Davey, Smith, G., Stansfeld, S., Patel, C., North, F., Head, J., White, I., Brunner, E., \& Feeney, A. (1991). Health inequalities among British civil servants: The Whitehall II study. Lancet, 337, 1387-1393.

Marmot, M. G., Shipley, M. J., \& Rose, G. (1984). Inequalities in death-specific explanations of a general pattern? Lancet, 1, 1003-1006.

McCally, M., Haines, A., Fein, O., Addington, W., Lawrence, R. S., \& Cassel, C. K. (1998). Poverty and ill health: Physicians can, and should, make a difference. Annals of Internal Medicine, 129, 726-733 (Review) (57 refs).

Page, R. M., Cole, G. E., \& Timmreck, T. C. (1995). Basic epidemiological methods and biostatistics. A practical guidebook. Sudbury, MA: Jones and Bartlett.

Ross, H. L. (1995). Housing code enforcement as law in action. Law and Policy, 17, 133-160.

Sallis, J. F., \& Owen, N. (1996). Ecological models. San Francisco: Jossey-Bass Publishers.

Sampson, R. J., \& Groves, W. B. (1989). Community structure and crime: Testing social-disorganization theory. American Journal of Sociology, 94, 774-802.

Sampson, R. J., \& Laub, J. H. (1990). Crime and deviance over the life course: The salience of adult social bonds. American Sociological Review, 55, 609-627.

Sampson, R. J., Raudenbush, S. W., \& Earls, F. (1997). Neighborhoods and violent crime: A multilevel study of collective efficacy. Science, 277, 918-924.

Shaw, M., David, G., Danny, D., Richard, M., \& Davey Smith, G. (2000). Increasing mortality differentials by residential area level of poverty: Britain 1981-1997. Social Science \& Medicine, 51, 151-153.

Welin, L., Larsson, B., Svardsudd, K., Tibblin, B., \& Tibblin, G. (1991). Social networks and activities in relation to mortality from cardiovascular diseases: A 12-year follow-up of the study of men born in 1913 and 1923. Journal of Epidemiology and Community Health, 45, 127-132.

Yankelovich, P. (1995). Survey of barriers to physical activity and healthy eating habits. In American Dietetic Association Conference, Chicago.

Yeracaris, C. A., \& Kim, J. H. (1978). Socioeconomic differentials in selected causes of death. American Journal of Public Health, 68, 342-351. 